\title{
Do unnecessary spectacles make the eyes worse
}

Children require spectacles for optical reasons-for the correction of important refractive errors (hypermetropia, myopia, and astigmatism). Refractive errors in children are detected usually by routine school vision tests, by parents or teachers noticing visual difficulties such as screwing up of the eyelids or uneven educational progress, or simply by knowledge of a family history of refractive errors.

There is also a therapeutic need for spectacles in the treatment of strabismus when there is a close link between accommodation and over convergence of the eyes. By relaxing the accommodation with convex (or positive) spectacle lenses the convergent strabismus can also be satisfactorily 'relaxed' or controlled. The need for these therapeutic spectacles (sometimes bifocals) gradually lessens during childhood and they may often be abandoned at puberty.

Spectacles are an emotive issue among parents and teachers. There is still a generally held belief that spectacles in some way 'cure' or 'treat' eye disease and children's eyesight will be harmed by not wearing correct spectacles. Of course spectacles play no part in the treatment of eye disease except for their therapeutic value in accommodation linked convergent strabismus.

Despite claims that refractive errors are environmentally induced-such as excessive close work 'causing' myopia-the overwhelming evidence is that refractive errors are genetically determined and alter during the period of growth in childhood. As a rule myopia increases, hypermetropia and astigmatism become less, and these changes are related to growth of the eye.

The detection of a refractive error does not necessarily mean the child has to wear spectacles. Small refractive errors do not really justify correction because the child observes no practical benefit, and spectacles become an unnecessary source of anxiety and conflict between parent and child.
Where there is an important refractive error (for example where uncorrected visual acuities are no better than $6 / 12$ binocularly) then spectacles are likely to be worn fairly happily by the child because he sees the immediate benefit to his visual acuity, and his developmental and educational progress is therefore enhanced. Children usually resist spectacles when they are prescribed for minor or uniocular refractive errors.

There is no evidence that spectacles influence the development of a refractive error. The natural increase in myopia, for example, where stronger spectacles are required every year or so may erroneously give rise to the impression of cause and effect. Where spectacles are prescribed for minor refractive errors in the mistaken belief they are responsible for symptoms such as headaches or academic failure, they cause no actual harm to the eyesight as far as is known. There may be a temporary reduction in accommodation but this is quickly rectified once the spectacles are left off. Spectacles which are too strong in myopic children may cause a spasm of accommodation but if time is taken for a careful cyclopłegic refraction to be carried out this mistake should not occur.

Prescribing spectacles is not simply a matter of measuring dioptres, as factors such as family compliance, cultural attitudes, behaviour abnormalities, and aptitude all have some bearing. Incorrect spectacles may cause some worsening of the eyes only if a squint is present as well, but for simple refractive errors alone it seems no harmful effects occur in the long term.

J L KENNERLEY BANKES St Mary's Hospital, Praed Street, London W2 $1 N 7$ 